gestation the Abdominal circumference, TF, FAST and EFW centile were all found to be statistically significant predictors of birthweight. Using backwards stepwise linear regression to find the optimal multivariate model for predicting birthweight a combination of EFW centile and TF were found to be the best predictors. At 37 weeks optimal multivariate model for BW prediction was EFW centile, FAST and TF. The results reveal acceptable reproducibility for fetal mid thigh muscle and fat for a single operator and between operators.

Conclusion This prospective study provides reference ranges for fetal mid thigh fat and muscle throughout gestation in fetuses with a normal growth velocity. The inclusion of fetal mid thigh fat in the birthweight algorithm improves the predictive power of birthweight estimation at 28 weeks and 37 weeks. This information is important to explore the role of fetal mid thigh in the detection of fetal IUGR at point estimations of EFW within normal centiles.

\section{PF.13 FETAL ECHOGENIC BOWEL: AN 18-YEAR REVIEW FROM THE WESSEX REGION}

doi:10.1136/archdischild-2013-303966.025

'S Walker, ${ }^{1} \mathrm{~A}$ Kermack, ${ }^{2} \mathrm{D}$ Wellesley, ${ }^{1} \mathrm{R}$ Parasuraman. ${ }^{1}$ Wessex Fetal Medicine Unit, Southampton, UK; ${ }^{2}$ Wessex Clinical Genetics Service, Southampton, UK

Background Echogenic bowel is a non-specific marker on antenatal ultrasound and is associated with a variety of underlying diagnoses and adverse fetal outcomes.

Objectives To evaluate fetal outcomes where echogenic bowel was identified on second trimester antenatal ultrasound.

Method Retrospective study of cases of echogenic bowel identified from the Fetal Medicine and Wessex Antenatally Detected Anomalies (WANDA) register between 1994 and 2012.

Results A total of 471 cases of echogenic bowel were identified over the 18-year period, of which 401 (85\%) were isolated.

Outcomes were available for $367(91.5 \%)$ cases. $322(80.2 \%)$ pregnancies resulted in a live birth, $21(5.2 \%)$ had termination of pregnancy and $19(4.7 \%)$ were complicated by intra-uterine demise. There were four $(1.0 \%)$ neonatal deaths.

Post-natal diagnosis was available for 358 (89.2\%) cases, of which $259(72.3 \%)$ were healthy, live-born infants. Cystic fibrosis and congenital cytomegalovirus infection was reported in ten $(2.8 \%)$ and five $(1.4 \%)$ respectively. Chromosomal abnormalities were present in 21 (5.9\%), with Trisomy 21 the most prevalent 15 (4.2\%). Intrauterine growth restriction complicated 17 (4.8\%) pregnancies. There were seven cases of intestinal atresia (2\%).

Conclusions In our cohort the majority of cases had a good outcome and no postnatal abnormality. However the increased incidence of cystic fibrosis, chromosomal abnormalities and congenital infection highlights the importance of investigating this group. The prevalence of growth restriction advocates the consideration of serial growth ultrasound. These findings are useful in the counselling of parents and antenatal management following the identification of isolated echogenic bowel.

\section{PF.14 ISOLATED BORDERLINE FETAL CEREBRAL VENTRICULOMEGALY - ROLE OF MAGNETIC RESONANCE IMAGING (MRI)}

doi:10.1136/archdischild-2013-303966.026

${ }^{1} \mathrm{JW}$ Gabriel, ${ }^{1 \mathrm{R}}$ Dworkin, ${ }^{2} \mathrm{M}$ To, ${ }^{2} \mathrm{~S}$ Bower, ${ }^{2} \mathrm{~N}$ Zosmer, ${ }^{3} \mathrm{R}$ Akeolar, ${ }^{2} \mathrm{~N}$ Flack. ${ }^{1} \mathrm{King}$ 's College London, London, UK; ${ }^{2}$ King's College Hospital, London, UK; ${ }^{3}$ Medway Maritime Hospital, Medway, UK

Objective To examine the role of third trimester magnetic resonance imaging (MRI) in fetuses diagnosed with isolated borderline cerebral ventriculomegaly at the routine second trimester fetal anomaly scan.
Methods This was a retrospective cohort study of 159 fetuses with apparently isolated borderline ventriculomegaly (9-12 $\mathrm{mm}$ ) diagnosed at the routine second trimester ultrasound scan at a median of 22 (range 19-24) weeks' gestation and no additional findings at a repeat scan 6-8 weeks later. Follow up cerebral MRI was carried out at 28-34 weeks and the number of cases in which this investigation demonstrated abnormal findings was calculated. The patients were examined in a tertiary fetal medicine unit between 2005 and 2012

Results In 7 (4.4\%) of the 159 cases the MRI scan demonstrated findings not seen by ultrasound. These included partial agenesis of the corpus callosum $(n=2)$, delayed sulcation disorders $(n=1)$, heterotopia $(n=2)$, germinal matrix haemorrhage $(n=1)$, and destruction of the septum pellucidum $(n=1)$.

Conclusions In about 4\% of fetuses with apparently isolated borderline cerebral ventriculomegaly an MRI scan demonstrates potentially clinically significant pathological findings.

\section{PF.15 EXPECTANT MANAGEMENT OF MONOCHORIONIC DIAMNIOTIC TWINS WITH SELECTIVE INTRAUTERINE GROWTH RESTRICTION}

doi:10.1136/archdischild-2013-303966.027

K Flood, FM Breathnach, FM McAuliffe, M Geary, S Daly, JR Higgins, J Dornan, JJ Morrison, G Burke, S Higgins, P Dicker, E Tully, S Carroll, FD Malone. Perinatal Ireland Research Consortium, Dublin, Ireland

We sought to evaluate the outcomes in a cohort of MCDA twins with a diagnosis of selective intra-uterine growth restriction (sIUGR) who were managed expectantly.

This prospective multicenter cohort study recruited 1,028 unselected twin pairs over 2 years in Ireland. Monochorionic twins underwent fortnightly ultrasonographic surveillance from 16 weeks. The defining criterion for sIUGR was an estimated fetal weight less than the $10^{\text {th }}$ centile in one twin with an appropriately grown co-twin. Details of the prenatal course, delivery timing and perinatal outcomes were recorded.

Outcome data were recorded for $100 \%$ of the 1,001 twin pairs that completed the study ( $\mathrm{n}=200$ monochorionic). Five percent $(\mathrm{n}=10)$ of the MCDA twin pregnancies were diagnosed with sIUGR at a median gestation of 30 weeks (range 26 - 35 weeks). AEDF or REDF was identified in two of these cases. The median time interval from diagnosis to delivery was 36.8 days (range $3-$ 66 days) at a mean gestation of 34.2 weeks (range $26-37.9$ weeks) $70 \%$ of the affected twins were admitted to the NICU with a mean stay of 19 days. There were no perinatal mortalities recorded.

Our findings demonstrated excellent outcomes for our cohort of MCDA twins complicated by selective IUGR. There was no single IUFD and in turn there was no morbidity conferred to the appropriately grown co-twin. Close surveillance with regular ultrasonography and Doppler evaluation was essential and allowed continuation of the majority affected pregnancies to a late gestational age, thereby optimising outcome for both twins.

\section{PF.16 MATERNAL PLASMA AND AMNIOTIC FLUID CYTOKINES IN MONOCHORIONIC TWIN PREGNANCIES COMPLICATED BY TWIN-TO-TWIN TRANSFUSION SYNDROME}

doi:10.1136/archdischild-2013-303966.028

${ }^{1,2}$ CE Fox, ${ }^{3} \mathrm{GE}$ Lash, ${ }^{2} \mathrm{SJ}$ Pretlove, ${ }^{2} \mathrm{BC}$ Chan, ${ }^{1 R}$ Holder, ${ }^{1,3} \mathrm{MD}$ Kilby. ${ }^{1}$ University of Birmingham, Edgbaston, Birmingham, UK; 'irmingham Women's Hospital NHS Foundation Trust, Edgbaston, Birmingham, UK; ${ }^{3}$ Newcastle University, Newcastle upon Tyne, UK

Objectives To investigate the maternal plasma and amniotic fluid (AF) cytokine concentrations in twin pregnancies complicated by TTTS and the effects of fetoscopic laser ablation (FLA). 